\title{
Gynaecomastia in two men on stable antiretroviral therapy who commenced treatment for tuberculosis
}

\author{
Jeremy D. Kratz ${ }^{1}$, Ahmad Y. El-Shazly ${ }^{2}$, Santos G. Mambuque ${ }^{2}$, Elpidio Demetria ${ }^{3}$, Peter Veldkamp ${ }^{4}$, \\ Timothy S. Anderson
}

1. Department of Medicine, University of Wisconsin Hospital and Clinics, Madison, Wisconsin, USA

2. Catholic University of Mozambique, Beira, Mozambique

3. Centro de Saude Sao Lucas, Beira, Mozambique

4. Division of Infectious Diseases, University of Pittsburgh Medical Center, Pittsburgh, Pennsylvania, USA

5. Division of General Internal Medicine, University of California San Francisco, San Francisco, California, USA

Correspondence: Dr Timothy S. Anderson (Timothy.Anderson@ucsf.edu)

\begin{abstract}
Gynaecomastia is a common clinical presentation that varies from benign presentations in stages of human development to hormonal pathology, mainly due to hepatic dysfunction, malignancy, and adverse pharmacologic effects. We describe the development of significant bilateral gynaecomastia after starting treatment for pulmonary tuberculosis (TB) in two males with WHO stage III Human Immunodeficiency Virus (HIV) infection on stable antiretroviral regimens.

Emerging reports suggest that distinct hepatic impairment in efavirenz metabolism modulates oestrogenic activity, which may be potentiated by antituberculosis therapy. Clinical application includes early recognition of efavirenz-induced gynaecomastia, especially after commencing tuberculosis treatment. To avoid decreased adherence resulting from the distressing side effect of gynecomastia, transition to an alternative ART regimen over the course of tuberculosis treatment should be considered.
\end{abstract}

\section{Case presentations}

1. A 32-year-old man presented to an urban outpatient clinic in Beira, Mozambique with bilateral gynaecomastia. The patient had been diagnosed with World Health Organization (WHO) stage III HIV disease two years prior to presentation and was maintained on an antiretroviral therapy (ART) regimen containing tenofovir (TDF) $300 \mathrm{mg}$ daily, lamivudine (3TC) $300 \mathrm{mg}$ daily, and efavirenz (EFV) $600 \mathrm{mg}$ daily. Eight months prior to presentation, he developed cough and weight loss, at which time he was diagnosed with pulmonary tuberculosis (TB). Therapy included a two-month regimen of rifampin (R) $600 \mathrm{mg}$ daily, isoniazid (H) $300 \mathrm{mg}$ daily, pyrazinamide (Z) $1600 \mathrm{mg}$ daily, and ethambutol (E) 1100 $\mathrm{mg}$ daily, followed by four months of rifampin $600 \mathrm{mg}$ daily and isoniazid $300 \mathrm{mg}$ daily (HRZE protocol). Two months into therapy he noted progressive nontender bilateral breast fullness. He denied alcohol, tobacco, or herbal medication use. He reported taking ART medications only as directed, without dosage changes. Review of systems was negative for abdominal distention, melaena, headache, vision changes, decreased libido, erectile dysfunction, or galactorrhoea. On examination, the patient was a well-appearing male, afebrile, with normal vital signs. Chest exam was notable for bilateral nontender gynaecomastia, shown in Figure 1A. The remainder of the physical exam was normal, including an abdomen without organomegaly or ascites, skin without spider telangiectasia, normal male hair pattern, normal phallus, and testes without masses. Two months after completing TB therapy, his breasts remained enlarged without further progression.

2. A 68-year-old man presented to the same outpatient clinic with acute onset of dysuria and secondary concern for breast enlargement. The patient had been diagnosed with WHO stage III HIV disease seven years prior to presentation and was maintained on ART containing zidovudine (AZT) 300 $\mathrm{mg}$ twice daily, 3TC $150 \mathrm{mg}$ twice daily, and EFV $600 \mathrm{mg}$ daily. Two months prior to presentation, he had completed a 6-month course of HRZE therapy (dosing as previously noted) for pulmonary TB. His breast fullness developed
Figure 1: Chest examination two months after completion of tuberculosis treatment

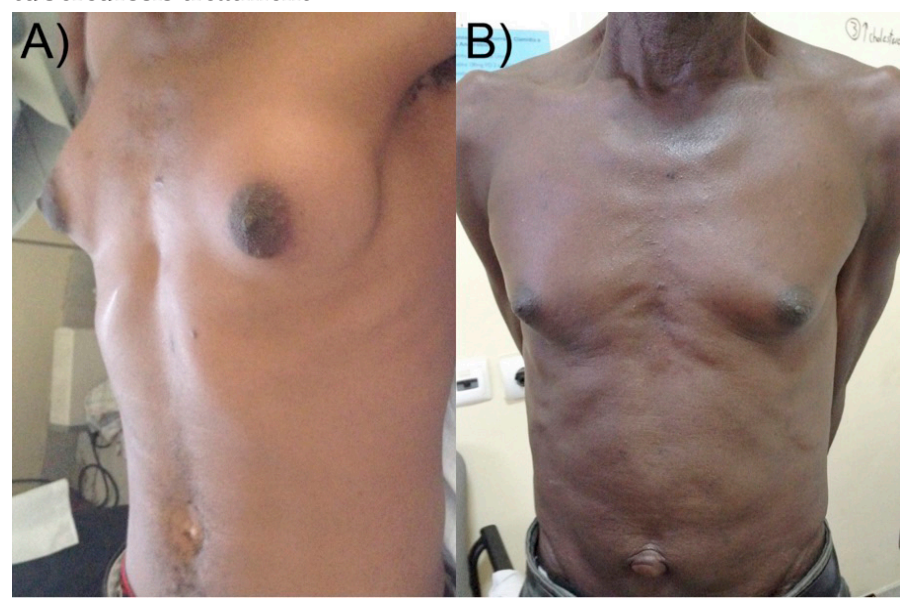

A) 34-year-old man in case 1

B) 68-year old-man in case 2

ten weeks after initiating HRZE therapy and persisted after completion. He denied fevers, chills, haematuria, abdominal pain, galactorrhoea, change in libido, or erectile dysfunction. On exam, he was a well-appearing male, afebrile, with normal vital signs. Findings on his chest exam (bilateral nontender gynaecomastia) are shown in Figure 1B. Rectal examination revealed an enlarged, tender, smooth prostate, without focal masses. The remainder of his exam revealed no hepatosplenomegaly, ascites, or spider telangiectasia, with normal phallus, testes without masses, and a normal male hair pattern. Given his dysuria and tender prostate, he was diagnosed and treated for prostatitis with a course of trimethoprim-sulfamethoxazole.

\section{Discussion}

Gynaecomastia is benign enlargement of the male breast, resulting from hyperplasia of underlying ductal tissue. The course of physiological gynaecomastia varies from reversible changes in puberty to permanent age-related manifestations. ${ }^{1}$ Other common aetiologies include endocrine disorders, hormone-secreting malignancies, severe hepatic dysfunction, and medication side effects. It was previously proposed 
Figure 2: Gynaecomastia as a result of synergy between medications and infectious disease

A
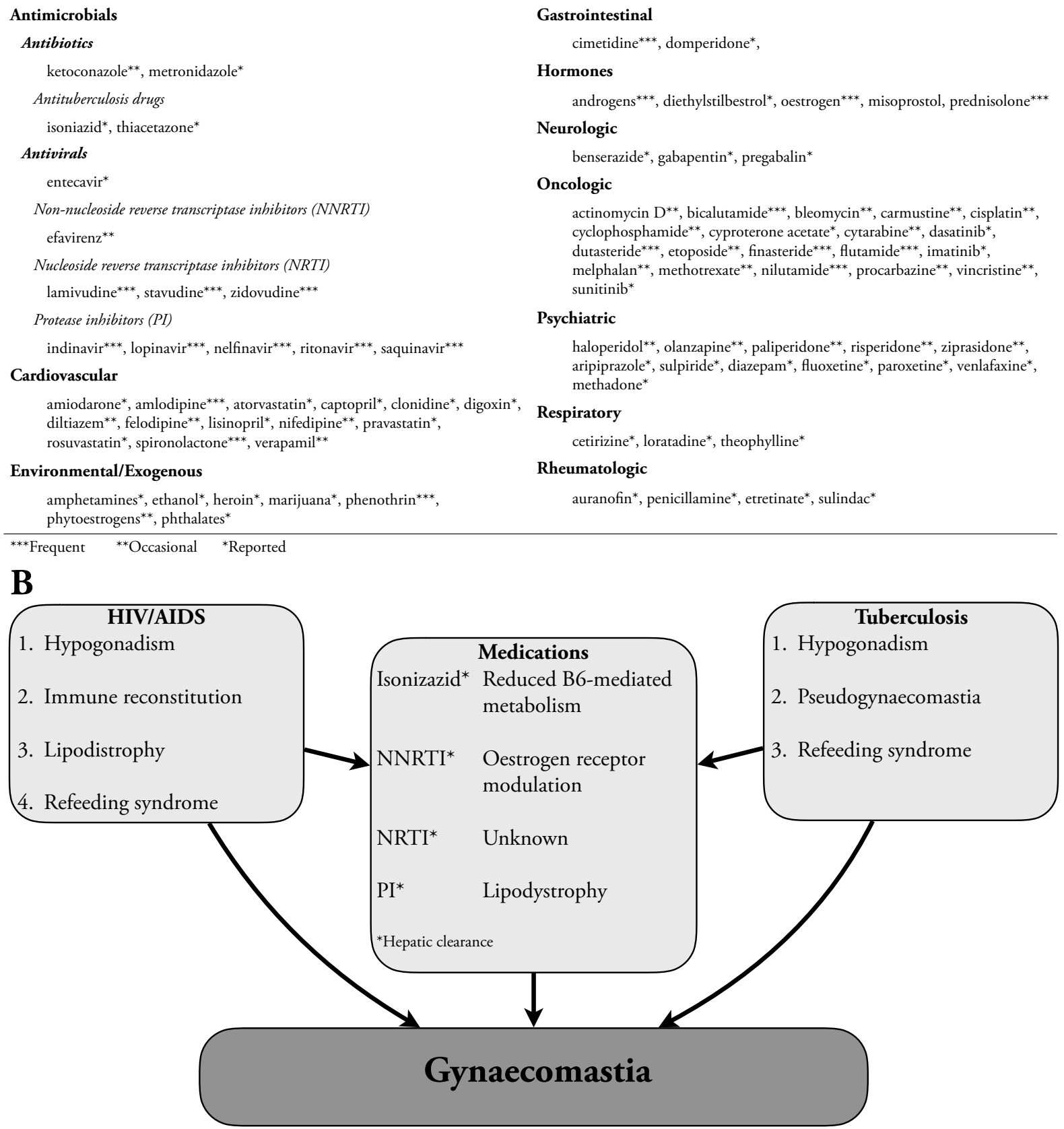

A) System-based medication review for gynaecomastia ${ }^{3}$

B) Synergistic mechanisms for gynaecomastia in patients with HIVIAIDS and tuberculosis

that EFV-induced gynaecomastia was mediated by either immune reconstitution or direct oestradiol-like effects, with mean clinical resolution at five months following removal of EFV. ${ }^{2}$ A previous case series reported six patients from Nigeria who developed gynaecomastia on EFV therapy; in five of these cases, the gynaecomastia resolved upon discontinuation of $\mathrm{EFV}^{3}$ A report from Malawi described two male patients who developed gynaecomastia over their course of ART containing EFV, including one patient who received concomitant HRZE therapy for $\mathrm{TB}^{4}{ }^{4}$ This case report builds upon these prior findings with the description of two additional cases of gynaecomastia from Mozambique. The two males infected with HIV were on stable ART containing EFV. They developed bilateral gynaecomastia after commencement of concurrent treatment for TB. While prior reports implicate EFV as the potential cause, this work builds on an evolving mechanistic understanding that highlights treatment interactions between HRZE and EFV.

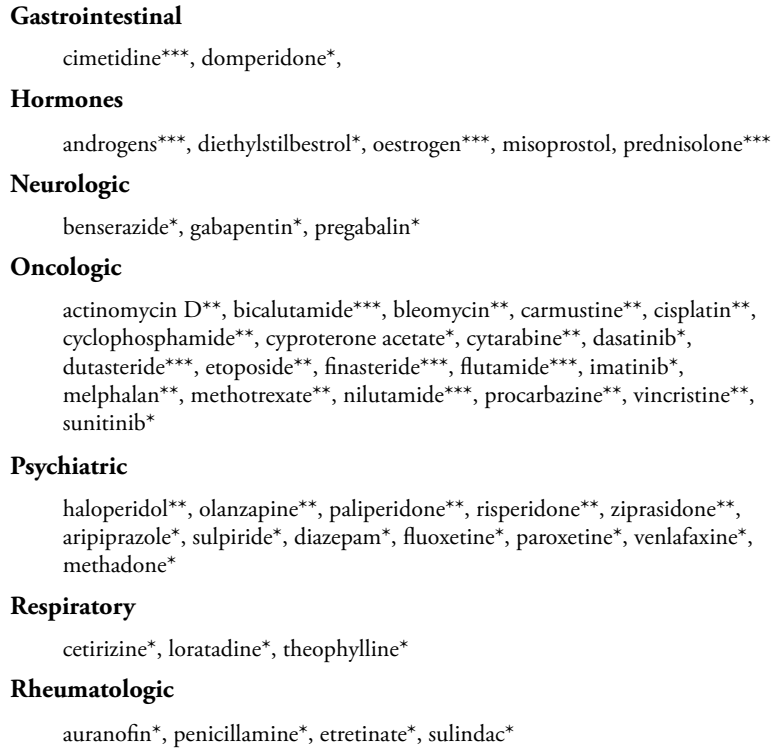

haloperidol**, olanzapine $e^{* *}$, paliperidone $e^{* *}$, risperidone $e^{* *}$, ziprasidone $e^{* *}$, aripiprazole*, sulpiride*, diazepam* ${ }^{*}$, fluoxetine*, paroxetine*, venlafaxine*, methadone*

Respiratory

cetirizine*, loratadine*, theophylline*

Rheumatologic

auranofin*, penicillamine*, etretinate*, sulindac*

Many agents have been implicated in the development of gynaecomastia, including ART and antimicrobial agents (see Figure 2A). ${ }^{5} \mathrm{HIV}$ and TB are both independent risk factors for hypogonadotrophic hypogonadism, with rates reported as high as $75 \%{ }^{6}$ and $73 \%{ }^{7}$ respectively, but these may have been influenced by cachexia in an era when effective HIV treatment was not available. No evidence of hypogonadism or lipodystrophy was found on the physical examinations of these patients. Although refeeding syndrome ${ }^{5}$ and immune reconstitution-induced gynaecomastia has been reported, ${ }^{8}$ these were unlikely causes, given the stable ART regimens (two and seven years) prior to initiation of therapy for TB.

Both histories favour potentiation of an otherwise stable ART regimen by the addition of HRZE. The time course, with progression over six months, suggested this potentiation by rifampin or isoniazid rather than ethambutol and pyrazinamide, which were given only in the first two months of HRZE. While there are few isolated isoniazid-induced cases 
of gynaecomastia reported, ${ }^{9}$ EFV may be more significant in potential causality, especially with the potentiating interaction with rifampin. Large prospective studies have documented an association between gynaecomastia and EFV and didanosine. ${ }^{10} \mathrm{EFV}$ is known to induce gynaecomastia by direct oestrogen receptor modulation, ${ }^{2}$ an effect that varies with ethnicity and weight. ${ }^{11}$ Mutations in CYP2B6 have been reported throughout Africa, ${ }^{12}$ as well as in Germany, ${ }^{13}$ resulting in impaired hepatic metabolism of EFV. Notably, these mutations have paradoxically augmented EFV levels with concurrent HRZE therapy. ${ }^{14}$

We report two patients with stable WHO stage III HIV disease, on EFV-containing ART regimens, who developed significant gynaecomastia during therapy for pulmonary TB. A prior report highlighted resolution of gynaecomastia in about $80 \%$ of patients on EFV-containing ART after change in therapy. ${ }^{8}$ Early recognition of gynaecomastia may prompt an early switch from an EFV-containing ART regimen to prevent worsening and effect reversal of this condition. Prior work has highlighted an algorithmic approach to managing gynaecomastia, including first identifying benign developmental aetiologies, and a recommendation for removal of potential offending agents. ${ }^{15} \mathrm{While}$ gynaecomastia may be clinically benign, it can be stigmatising for patients, who may compromise their medication adherence because of these concerns.

\section{References}

1. Narula HS, Carlson HE. Gynaecomastia--pathophysiology, diagnosis and treatment. Nat Rev Endocrinol. 2014;10(11):684-698.

2. Jover F, Cuadrado JM, Roig P, Rodriguez M, Andreu L, Merino J. Efavirenz-associated gynecomastia: report of five cases and review of the literature. Breast J. 2004;10(3):244-246.

3. Agbaji OO, Agaba PA, Ekeh PN, et al. Efavirenz-induced gynaecomastia in HIV-infected Nigerian men: A report of six cases. 2011.
4. Kwekwesa A, Kandionamaso C, Winata N, et al. Breast enlargement in Malawian males on the standard first-line antiretroviral therapy regimen: Case reports and review of the literature. Malawi Med J. 2015;27(3):115117.

5. Bowman JD, Kim H, Bustamante JJ. Drug-induced gynecomastia. Pharmacotherapy. 2012;32(12):1123-1140.

6. Dobs AS, Dempsey MA, Ladenson PW, Polk BF. Endocrine disorders in men infected with human immunodeficiency virus. Am J Med. 1988;84(3 Pt 2):611-616.

7. Post FA, Soule SG, Willcox PA, Levitt NS. The spectrum of endocrine dysfunction in active pulmonary tuberculosis. Clin Endocrinol (Oxf). 1994;40(3):367-371.

8. Qazi NA, Morlese JF, King DM, Ahmad RS, Gazzard BG, Nelson MR. True gynaecomastia, another manifestation of immune reconstitution disease? Int J STD AIDS. 2002;13(1):59-65.

9. Khan A, Agarwal R. Isoniazid related gynecomastia: Description of a case and systematic review of literature. Lung India. 2012;29(2):189-191.

10. Mira JA, Lozano F, Santos J, et al. Gynaecomastia in HIV-infected men on highly active antiretroviral therapy: association with efavirenz and didanosine treatment. Antivir Ther. 2004;9(4):511-517.

11. Stohr W, Back D, Dunn D, et al. Factors influencing efavirenz and nevirapine plasma concentration: effect of ethnicity, weight and comedication. Antivir Ther. 2008;13(5):675-685.

12. Gross R, Aplenc R, Tenhave T, et al. Slow efavirenz metabolism genotype is common in Botswana. J Acquir Immune Defic Syndr. 2008;49(3):336-337.

13. Wyen C, Hendra H, Vogel M, et al. Impact of CYP2B6 983T $>C$ polymorphism on non-nucleoside reverse transcriptase inhibitor plasma concentrations in HIV-infected patients. J Antimicrob Chemother. 2008;61(4):914-918.

14. Kwara A, Lartey M, Sagoe KW, Court MH. Paradoxically elevated efavirenz concentrations in HIV/tuberculosis-coinfected patients with CYP2B6 516TT genotype on rifampin-containing antituberculous therapy. AIDS. 2011;25(3):388-390.

15. Morcos RN, Kizy T. Gynecomastia: when is treatment indicated? J Fam Pract. 2012;61(12):719-725. 